\title{
Antenna Selection for Unitary Space-Time Modulation over Correlated Rayleigh Channels
}

\author{
Mahdi Hajiaghayi and Chintha Tellambura \\ Department of Electrical and Computer Engineering \\ University of Alberta \\ Edmonton, AB, Canada, T6G 2V4 \\ Email:\{mahdih,chintha\}@ece.ualberta.ca
}

\begin{abstract}
In [1], antenna selection for multiple antenna systems that employ unitary space-time (ST) signaling has been studied under the assumption of an independent Rayleigh fading channel. In this paper, the performance of such a system is investigated for spatially correlated-fading channels. The channel state information (CSI) is not known at the receiver nor at the transmitter. Antenna selection is thus performed at the receiver based on the instantaneous received signal power. By deriving the Chernoff bound on the pairwise error probability (PEP), we quantify the effects of channel correlation on the diversity order and coding gain at high signal-to-noise ratio (SNR). Analytical results indicate that the full diversity order is preserved in such a channel as long as the unitary signals are full rank. However, spatial correlations result in a loss of the coding gain.
\end{abstract}

\section{INTRODUCTION}

$\mathbf{U}$ NITARY space time modulation (USTM) is a multipleinput multiple-output (MIMO) signaling technique for the case where neither the transmitter nor the receiver knows the channel state information (CSI) [2], [3]. In this case, USTM performs well and achieves full spatial diversity gains over the fast-fading channels. Most previous USTM studies assumed that all the available antennas are utilized for signal transmission and reception [3], [4]. However, since each active transmit/receive antenna pair requires an RF (radio frequency) chain, which is expensive, antenna selection, where a subset of all available antennas are selected at the transmitter and/or receiver, has been extensively considered [5], [6]. Nevertheless, most previous antenna selection studies focus on coherent multi-antenna systems in which perfect CSI is available at the receiver [7].

The only study that dealt with USTM and receive antenna selection (RAS) without CSI is [1]. However, it considered only the case of independent Rayleigh-fading channels and showed that the diversity order is preserved if USTM and RAS are used. In practical systems, insufficient antenna spacing, angle spread or the lack of rich scattering may cause spatial correlation among antennas, particularly at the transmitter side [8]. Moreover, channel measurements reveal that in some propagation environments, a fixed (possibly line of sight (LoS)) component is present, [9]. In this case, the mean of the channel matrix is not zero and the Ricean fading model is used to formulate the channel matrix,

In this paper, the analysis of [1] is extended to the correlated-fading channels. RAS is based on the instantaneous received signal power. The optimal decoders based on this selection rule is presented and the Chernoff bound on the pairwise error probability (PEP) is derived. The channel correlation is modeled by using the exponential correlation model. Extension of our work for other correlation models and Ricean channels is presented in a forthcoming journal version of this paper. Our analytical results indicate that the full diversity is preserved in correlated Rayleigh channels when USTM and RAS are employed. However, as might be expected, the spatial correlations result in a loss of the coding gain.

The reminder of this paper is organized as follows: Section II describes the system model and presents a brief overview of USTM and differential USTM. In Section III, we present the selection rule and the Chernoff bound on PEP for the correlated-fading channel. The exponential correlation model is defined, and the diversity order and coding gain associated with that model are derived. The Chernoff-bound expression is simplified for a $2 \times 2$ MIMO system with single antenna selection. The numerical results are shown in Section IV and concluding remarks are given in Section V.

Notation: the Hermitian, transpose, trace, determinant and Frobenius norm of matrix $\mathbf{A}$ is denoted by $\mathbf{A}^{\mathrm{H}}, \mathbf{A}^{\mathrm{T}}, \operatorname{tr}(\mathbf{A})$, $\operatorname{det}(\mathbf{A}),\|\mathbf{A}\|_{F}$, respectively. A circularly symmetric complex Gaussian random variable (RV) with mean $\mu$ and variance $\sigma^{2}$ is denoted by $z \sim \mathcal{C N}\left(\mu, \sigma^{2}\right)$. $\max _{n}^{J} X_{n}$ selects the $J$ largest $X_{n}$ 's.

\section{SySTEM Model AND USTM SCheme}

Consider a MIMO system with $M$ transmit and $N$ receive antennas signaling over a frequency flat-fading channel. The channel coefficient between the $i$-th transmit antenna and the $j$-th receive antenna is denoted by $h_{i j} \sim \mathcal{C N}(0,1)$. For blocks of $T$ consecutive time samples, the input-output relationship is [2]

$$
\mathbf{Y}_{\tau}=\sqrt{\frac{\rho}{M}} \mathbf{S}_{\tau} \mathbf{H}+\mathbf{W}_{\tau}
$$

where $\mathbf{Y}_{\tau}=\left[\mathbf{y}_{1}, \ldots, \mathbf{y}_{N}\right]$ is a $T \times N$ complex received signal matrix, $\mathbf{S}_{\tau}$ is a $T \times M$ complex transmitted signal matrix and $\mathbf{W}_{\tau}$ denotes a $T \times N$ additive noise matrix with i.i.d. $\mathcal{C N}(0,1)$ elements, and the block-time index is $\tau$. The transmitted energy on all $M$ antennas at any time slot is 
normalized to unity ensuring that $\rho$ is the average SNR per receiver.

The $M \times N$ random channel-matrix $\mathbf{H}$ consists of a fixed component and a random component. It is generally modeled as

$$
\mathbf{H}=\sqrt{\frac{K}{K+1}} \overline{\mathbf{H}}+\sqrt{\frac{1}{K+1}} \mathbf{R}_{T}^{1 / 2} \mathbf{H}_{w} .
$$

where the first and second terms of (2) are the mean (line of sight (LoS) component) and the diffuse part of the channel, respectively. In (2) $\mathbf{H}_{w}$ is an $M \times N$ matrix composed of i.i.d. $\mathcal{C N}(0,1)$ RVs and $\mathbf{R}_{T}$ is an $M \times M$ positive definite matrix denoting the spatial transmit correlation. The matrix $\mathbf{R}_{T}$ is normalized so that $\left[\mathbf{R}_{T}\right]_{i, i}=1(i=1,2, \ldots, M)$, resulting in $E\left\{\left|h_{i, j}\right|^{2}\right\}=1$. In this paper, the correlated channel model without a LOS component $(K=0)$ is considered. USTM employs a codebook of matrix signals $\mathcal{V}$ at both the transmitter and receiver sides, which contains $L$ unitary matrix space-time signals [2] $\mathbf{S}_{1}=\sqrt{T} \boldsymbol{\Phi}_{1}, \ldots, \mathbf{S}_{L}=\sqrt{T} \boldsymbol{\Phi}_{L}$, where the $T \times M$ matrix $\boldsymbol{\Phi}_{l}$ satisfies $\boldsymbol{\Phi}_{l}^{\mathrm{H}} \boldsymbol{\Phi}_{l}=I_{M}$ for $0 \leq l<L$. To transmit a data sequence of integers $z_{1}, z_{2}, \ldots$ with $z_{\tau} \in\{0, \ldots, L-1\}$, each $z_{\tau}$ is mapped to a distinct unitary matrix signal $\boldsymbol{\Phi}_{z_{\tau}}$ drawn from the codebook $\mathcal{V}$ and ultimately $\mathbf{S}_{\tau}=\sqrt{T} \boldsymbol{\Phi}_{z_{\tau}}$ is transmitted over multiple antennas. In differential USTM [10], $d_{z_{\tau}}$ is mapped to an $M \times M$ differential unitary matrix $\mathbf{V}_{z_{\tau}}$ and transmitted signal $\mathbf{S}_{\tau}$ at time $\tau$ is generated by

$$
\mathbf{S}_{\tau}= \begin{cases}\mathbf{V}_{z_{\tau}} \mathbf{S}_{\tau-1} & \tau=1,2, \ldots, \\ \mathbf{I}_{M} & \tau=0\end{cases}
$$

From [10], differential USTM can be viewed as a special case of the general USTM scheme by defining an equivalent $T \times M$ unitary matrix $\boldsymbol{\Phi}_{z_{\tau}}$ of the form $\boldsymbol{\Phi}_{z_{\tau}}=$ $1 / \sqrt{2}\left[\mathbf{I}_{M}, \mathbf{V}_{z_{\tau}}\right]^{T}$, where $T=2 M$. As a result, to cover the general case, only the USTM scheme is considered.

\section{ERror ProbabiLITY OF USTM RAS IN CORRELATED FADING}

In this section, the performance of the USTM and RAS in a correlated channel is analyzed. The antenna selection rule and the decoding algorithm are described, and based on the Chernoff bound on the PEP, the diversity order and coding gain are derived.

The selection rule here is a commonly used way of selecting a receive antenna which is implemented by a simple maximum-norm detection circuit. This rule does not require the receiver to know the CSI or even the correlation matrix. The rule is that the $J$ antennas whose received signal norms are the largest among all the antennas are selected [1]; i.e,

$$
\left[n_{1}, \cdots, n_{J}\right]=\arg \max _{n=1,2, \cdots, N}^{J} z_{n},
$$

where $z_{n}=\left\|\mathbf{y}_{n}\right\|^{2}$. Based on the argument in [1], the following inequality for the Chernoff bound on the PEP of mistaking $\boldsymbol{\Phi}_{l}$ for $\boldsymbol{\Phi}_{l^{\prime}}$ can be derived that changes to equality when $J=1$;

$P_{\mathrm{CB}}(\mu) \leq \frac{\pi^{-T J} N !}{2 \operatorname{det}\left(\mathbf{R}_{l}\right)^{J}(N-J) ! J ! J} \sum_{i=1}^{J} \int_{\mathcal{C}^{T}} F_{z}\left(\left\|\mathbf{y}_{i}\right\|^{2}\right)^{N-J} \times \exp$

$\left(-\sum_{k=1}^{J} \mathbf{y}_{k}^{\mathrm{H}}\{\underbrace{\mu \mathbf{R}_{l^{\prime}}^{-1}+(1-\mu) \mathbf{R}_{l}^{-1}}_{\boldsymbol{\Omega}(\mu)}\} \mathbf{y}_{k}\right) d \mathbf{y}_{1} \cdots d \mathbf{y}_{J}$,

where $C^{T}$ is the T-dimensional complex space and $0 \leq \mu \leq 1$ is a free parameter that is chosen to minimize $P_{\mathrm{CB}}(\mu)$. In (5), $F_{z}($.$) denotes the cumulative density function (CDF) of \|\mathbf{y}\|^{2}$, and $\mathbf{R}_{l}$ and $\mathbf{R}_{l^{\prime}}$ are the $T \times T$ covariance matrices conditioned on $\boldsymbol{\Phi}_{l}$ and $\boldsymbol{\Phi}_{l^{\prime}}$ transmitted, respectively. The covariance matrix $\mathbf{R}_{l}$ can be written as

$$
\mathbf{R}_{l}=\mathbf{I}_{T}+\frac{\rho T}{M} \boldsymbol{\Phi}_{l} \mathbf{R}_{T} \boldsymbol{\Phi}_{l}^{\mathrm{H}} .
$$

Note that each antenna at the receiver observes independently correlated-fading gains from the transmitter antennas provided that no correlation between the receive antennas exists. As a result, all the columns of the received signal $\mathbf{Y}$ are i.i.d.. Since the received signal vector at each receiver, say receiver $i$, is a Gaussian RV vector with not necessarily zero mean, $F_{z}\left(\left\|\mathbf{y}_{i}\right\|^{2}\right)$ is also called the CDF of the quadratic form of Gaussian RVs. By using the matrix inversion and determinant lemmas in [2], one can derive

$$
\mathbf{R}_{l}^{-1}=\mathbf{I}_{T}-\boldsymbol{\Phi}_{l}\left(\mathbf{I}_{M}+\left(\frac{\rho T}{M} \mathbf{R}_{T}\right)^{-1}\right)^{-1} \boldsymbol{\Phi}_{l}^{\mathrm{H}}
$$

and

$$
\operatorname{det}\left(\mathbf{R}_{l}\right)=\operatorname{det}\left(\mathbf{I}_{M}+\frac{\rho T}{M} \mathbf{D}\right),
$$

where the diagonal matrix $\mathbf{D}=\operatorname{diag}\left\{\lambda_{1}, \ldots, \lambda_{M}\right\}$ is obtained from the singular value decomposition of $\mathbf{R}_{T}$.

Using the similar technique as in [1] and considering the fact that $F_{z}\left(\|\mathbf{y}\|^{2}\right)$ is independent of the transmitted signal $\mathbf{\Phi}_{l}$ (this independence will be proved shortly), the ML detection is expressed as

$\mathbf{\Phi}_{\mathrm{ML}}=\arg \max _{\mathbf{\Phi}_{l} \in\left\{\boldsymbol{\Phi}_{1}, \ldots, \boldsymbol{\Phi}_{L}\right\}} \operatorname{tr}\left\{\hat{\mathbf{Y}}^{\mathrm{H}} \mathbf{\Phi}_{l}\left(\mathbf{I}_{M}+\frac{M}{\rho T} \mathbf{D}^{-1}\right)^{-1} \boldsymbol{\Phi}_{l}^{\mathrm{H}} \hat{\mathbf{Y}}\right\}$,

where $\hat{\mathbf{Y}}=\left[\mathbf{y}_{n_{1}}, \cdots, \mathbf{y}_{n_{J}}\right]$. If we assume that the receiver knows the correlation matrix, the ML detector requires searching over the codebook $\mathcal{V}$ in order to find the optimum signal by maximizing (9). Although the RAS criterion in (4) does not require the correlation matrix $\mathbf{R}_{T}$, this information is needed in (9) to extract the most likely transmitted signal. One popular transmit-correlation model of $\mathbf{R}_{T}$ is exponential correlation model. This model, which is a $M \times M$ matrix with entries

$$
\left[\mathbf{R}_{T}\right]_{i, j}=\gamma^{|i-j|}
$$

may hold for the practical case of the equispaced linear array of antennas [11]. The authors of [12] have proved that the matrix (10) has $M$ distinct eigenvalues that can be expressed as the functions of the correlation coefficient $\gamma$. 
First, we will briefly outline the derivation procedure. To compute $P_{\mathrm{CB}}$ in (5), $F_{z}\left(\|\mathbf{y}\|^{2}\right)$ is required. Since the diversity order and coding gain are meaningful only in the asymptotically high SNR region, to find them, the Chernoff bound on the error probability is derived as a power series of the SNR. The diversity order and coding gain are then extracted from this power series. For example, at asymptotically high SNRs, if the following relation [13],

$$
P_{\mathrm{CB}}=\left(G_{c} \rho\right)^{-G_{d}}+o\left(\rho^{-G_{d}}\right),
$$

holds for the error probability or the Chernoff bound, then $G_{d}$ and $G_{c}$ represent the diversity order and coding gain, respectively. In order to evaluate $P_{\mathrm{CB}}$ at high SNR $(\rho \rightarrow \infty)$, the dominant term in the power series expansion of $F_{z}(a)$ in terms of $\rho$ is required. This CDF can be obtained by inverting the Laplace transform of the moment generating function (MGF) of $z_{i}=\left\|\mathbf{y}_{i}\right\|^{2}$ given by [14, page 595]. With the use of partial fractions, it is straightforward to show that the characteristic function of $z_{i}$ is obtained as

$$
\begin{aligned}
G(s) & =\frac{1}{(1+s)^{T-M} \prod_{i=1}^{M}\left[1+s(\underbrace{\frac{\rho T}{M} \lambda_{i}+1}_{r_{i}})\right]} \\
& =\sum_{k=1}^{T-M} \frac{A_{k}}{(1+s)^{k}}+\sum_{k=1}^{M} \frac{B_{k}}{\left[1+s(\underbrace{\frac{\rho T}{M} \lambda_{k}+1}_{r_{k}})\right]},
\end{aligned}
$$

where

$$
\begin{aligned}
& A_{T-M-k}=\left.\frac{1}{k !} \frac{\partial^{k}}{\partial s^{k}}\left(\prod_{i=1}^{M}\left[1+s r_{i}\right]^{-1}\right)\right|_{s=-1} \\
& B_{k}=\left(\frac{1}{1-\frac{1}{r_{k}}}\right)^{T-M} \prod_{i=1, i \neq k}^{M}\left(1-\frac{r_{i}}{r_{k}}\right)^{-1} .
\end{aligned}
$$

The PDF of $z_{i}$ can be obtained by taking the inverse Laplace transform of (12) as follows:

$$
\begin{aligned}
f_{z}(u) & =\mathcal{L}^{-1}\{G(s)\} \\
& =\sum_{k=1}^{T-M} A_{k} \frac{1}{(k-1) !} u^{k-1} e^{-u}+\sum_{k=1}^{M} \frac{B_{k}}{r_{k}} e^{-u / r_{k}} .
\end{aligned}
$$

By integrating (14), the CDF of $z_{i}$ is obtained as

$$
F_{z}(a)=\sum_{k=1}^{T-M} A_{k}\left(1-e^{-a} \sum_{i=0}^{K-1} \frac{a^{i}}{i !}\right)+\sum_{k=1}^{M} B_{k}\left(1-e^{-a / r_{k}}\right) .
$$

Notice that the CDF expression of $F_{z}(a)$ in (15) is independent of the transmitted signal $\boldsymbol{\Phi}_{l}$. By inserting (13b) into (15) and after some manipulations, the second term of the right side of
(15) approaches to

$$
\begin{aligned}
\sum_{k=1}^{M} B_{k}\left(1-e^{-a / r_{k}}\right) & \rightarrow-\sum_{j=1}^{\infty} \frac{(-a M)^{j}}{(\rho T)^{j} j !} \\
& \times \sum_{k=1}^{M} \lambda_{k}^{-j} \prod_{i=1, i \neq k}^{M}\left(1-\frac{\lambda_{i}}{\lambda_{k}}\right)^{-1}
\end{aligned}
$$

when $\rho \rightarrow \infty$. Upon cursory examination of (16), one might conclude that the dominant term occurs at $j=1$ as $\rho \rightarrow \infty$. However, the most dominant term in the power series of $F_{z}(a)$ is the term $\rho^{-M}$ because the coefficient of any power term $\rho^{j}$, $j<M$, is zero.

Theorem 1: For any M distinct eigenvalues $\lambda_{1}, \ldots, \lambda_{M}$ and $0<j<M$, the following holds:

$$
\sum_{k=1}^{M}\left(\lambda_{k}\right)^{-j+M-1} \prod_{i=1, i \neq k}^{M}\left(\lambda_{k}-\lambda_{i}\right)^{-1}=0 .
$$

Proof: With the common denominator $\prod_{1 \leq i<j \leq M}\left(\lambda_{i}-\lambda_{j}\right)$, the numerator of the left side of (17) would be

$$
\begin{gathered}
\sum_{k=1}^{M}(-1)^{k-1} \lambda_{k}^{-j+M-1} \prod_{\substack{1 \leq i<j \leq M \\
i, j \neq k}}\left(\lambda_{i}-\lambda_{j}\right)= \\
\operatorname{det}\left[\begin{array}{cccc}
\lambda_{1}^{-j+M-1} & \lambda_{2}^{-j+M-1} & \ldots & \lambda_{M}^{-j+M-1} \\
1 & 1 & \ldots & 1 \\
\lambda_{1} & \lambda_{2} & \cdots & \lambda_{M} \\
\lambda_{1}^{2} & \lambda_{2}^{2} & \cdots & \lambda_{M}^{2} \\
\vdots & \vdots & \ldots & \vdots \\
\lambda_{1}^{M-2} & \lambda_{2}^{M-2} & \ldots & \lambda_{M}^{M-2}
\end{array}\right] .
\end{gathered}
$$

The right side of (18) arises from the use of the rotational properties of the Vandermonde Matrix (see Section 6.1 [15]) and the general definition of the determinant as well. The left side of (18) is now equal to zero for all integers $1 \leq j \leq M-1$ because if $j$ is in this interval, the introduced matrix (18) has two equal rows, so its determinant clearly must be zero.

Thus, the second term of (15) is expanded as

$$
\begin{gathered}
\sum_{k=1}^{M} B_{k}\left(1-e^{-a / r_{k}}\right)=-\frac{(-a M)^{M}}{\rho^{M} T^{M} M !} \sum_{k=1}^{M} \lambda_{k}^{-M} \prod_{\substack{i=1 \\
i \neq k}}^{M}\left(1-\frac{\lambda_{i}}{\lambda_{k}}\right)^{-1} \\
+o\left(\rho^{-M}\right) .
\end{gathered}
$$

Similarly, the dominant term of the first term of (15) is $\rho^{-M}$ as $\rho \rightarrow \infty$.

Theorem 2: For any integer $k$, and for large $\rho$, the following holds:

$$
\left.\frac{\partial^{k}}{\partial s^{k}}\left(\prod_{i=1}^{M}\left[1+s r_{i}\right]^{-1}\right)\right|_{s=-1}=C_{k} \rho^{-M}+o\left(\rho^{-M}\right),
$$

where $C_{k}$ is a constant value, independent of $\rho$, and uniquely determined for each $k$. In other words, the left side of (20) behaves as $C_{k} \rho^{-M}$ when $\rho$ is sufficiently large. 
Proof: See Appendix A.

In the high-SNR region, $F_{z}(a)^{N-J}$ can be approximated by retaining the first term in the power series expansion of $F_{z}(a)$; thus,

$$
\begin{gathered}
F_{z}(a)^{N-J}=\frac{1}{\rho^{M(N-J)}}\left[\sum_{k=1}^{T-M} \mathcal{D}_{k}\left(1-e^{-a} \sum_{i=0}^{K-1} \frac{a^{i}}{i !}\right)+a^{M} \mathcal{E}\right]^{N-} \\
+o\left(\rho^{-M(N-J)}\right),
\end{gathered}
$$

where

$$
\begin{aligned}
& \mathcal{D}_{k}=C_{T-M-k} \frac{(-1)^{T-M-k}}{(T-M-k) !} \\
& \mathcal{E}=\left(\sum_{k=1}^{M}(-1)^{M+1} \frac{M^{M}}{T^{M} M ! \lambda_{k}^{M}} \prod_{\substack{i=1 \\
i \neq k}}^{M}\left(1-\frac{\lambda_{i}}{\lambda_{k}}\right)^{-1}\right) .
\end{aligned}
$$

Therefore, by substituting (8) and (21) in (5), we obtain

$$
\begin{aligned}
& P_{\mathrm{CB}}(\mu) \leq \frac{N ! \prod_{i=1}^{M}\left(1+\frac{\rho T}{M} \lambda_{i}\right)^{-J}}{2 \pi^{T J}(N-J) ! J ! J} \sum_{i=1}^{J} \int_{\mathcal{C}^{T}}\left[e^{-\sum_{k=1}^{J} \mathbf{y}_{k}^{\mathrm{H}} \boldsymbol{\Omega}(\mu) \mathbf{y}_{k}}\right. \\
& \times \frac{1}{\rho^{M(N-J)}}\left(\sum_{k=1}^{T-M} \mathcal{D}_{k}\left(1-e^{-\mathbf{y}_{i}^{\mathrm{H}} \mathbf{y}_{i}} \sum_{i=0}^{K-1} \frac{\left(\mathbf{y}_{i}^{\mathrm{H}} \mathbf{y}_{i}\right)^{i}}{i !}\right)\right. \\
& \left.\left.+\left(\mathbf{y}_{i}^{\mathrm{H}} \mathbf{y}_{i}\right)^{M} \mathcal{E}\right)^{N-J}\right] d \mathbf{y}_{1} \cdots d \mathbf{y}_{J}+o\left(\rho^{-M N}\right) .
\end{aligned}
$$

From (7), $\boldsymbol{\Omega}(\mu)$ may be rewritten as

$$
\begin{aligned}
\boldsymbol{\Omega}(\mu) & =\mathbf{I}_{T}-\mu \boldsymbol{\Phi}_{l^{\prime}}\left(\mathbf{I}_{M}+\frac{M}{\rho T} \mathbf{R}_{T}^{-1}\right)^{-1} \boldsymbol{\Phi}_{l^{\prime}}^{\mathrm{H}}-(1-\mu) \boldsymbol{\Phi}_{l} \\
& \times\left(\mathbf{I}_{M}+\frac{M}{\rho T} \mathbf{R}_{T}^{-1}\right)^{-1} \boldsymbol{\Phi}_{l}^{\mathrm{H}} \Rightarrow \\
& \lim _{\rho \rightarrow \infty} \boldsymbol{\Omega}(\mu)=\mathbf{I}_{T}-\mu \boldsymbol{\Phi}_{l^{\prime}} \boldsymbol{\Phi}_{l^{\prime}}^{\mathrm{H}}-(1-\mu) \boldsymbol{\Phi}_{l} \boldsymbol{\Phi}_{l}^{\mathrm{H}} .
\end{aligned}
$$

We assume for all $\boldsymbol{\Phi}_{l}$ and $\boldsymbol{\Phi}_{l^{\prime}}$ drawn from the unitary constellation $\mathcal{V}, \operatorname{rank}(\boldsymbol{\Omega}(\mu))=T$ for all $l \neq l^{\prime}$ (i.e. the full diversity constellation). In the case of single antenna selection, by using the singular value decomposition $\boldsymbol{\Omega}(\mu)=$ $\mathbf{Q} \operatorname{diag}\left\{\alpha_{1}, \ldots, \alpha_{T}\right\} \mathbf{Q}^{\mathrm{H}}$ in (24) and changing the variables $x_{t}=\left|\bar{y}_{t}\right|^{2}$, where $\bar{y}_{t}$ is the $t$-th element of vector $\mathbf{Q}^{\mathrm{H}} \mathbf{y}$, the Chernoff bound on PEP from (23) is obtained. Therefore, the diversity and coding gain of USTM and RAS are

$G_{d}=M N$

$G_{c}=\left(\frac{N M^{M}}{2 T^{M} \operatorname{det}\left(\mathbf{R}_{\mathbf{T}}\right)} \int_{0}^{\infty} \cdots \int_{0}^{\infty}\left[e^{-\sum_{t=1}^{T} \alpha_{t} x_{t}}\left(\sum_{k=1}^{T-M} \mathcal{D}_{k}\right.\right.\right.$

$\left.\left.\times\left(1-e^{-\sum_{t=1}^{T} x_{t}} \sum_{i=0}^{k-1} \frac{\left(\sum_{t=1}^{T} x_{t}\right)^{i}}{i !}\right)+\left(\sum_{t=1}^{T} x_{t}\right)^{M} \mathcal{E}\right)^{N-1}\right]$

$\left.\times d x_{1} \cdots d x_{T}\right)^{-1 /(M N)}$.

We observe that for high SNR and full rank space-time codes, the full diversity order remains when a single antenna selected.
This result can be generalized to the multiple antenna selection case from Eq. (23). Depending on the determinant of $\mathbf{R}_{T}$, some loss in the coding gain occurs. The equations (25a) and (25b) can be more simplified for the case of a 2 by 2 MIMO system when the differential USTM scheme with $T=4$ is employed. Without providing the details of the proof, $P_{\mathrm{CB}}$ for such system is obtained as

$$
\begin{aligned}
& P_{\mathrm{CB}}=\frac{1}{16 \rho^{4}}\left(1-\gamma^{2}\right)^{-2} \int_{0}^{\infty} e^{-\sum_{t=1}^{4} \alpha_{t} x_{t}}\left[2\left(1-e^{-\sum_{t=1}^{4} x_{t}}\right)\right. \\
& \left.+1-\left(1+\sum_{t=1}^{4} x_{t}\right) e^{-\sum_{t=1}^{4} x_{t}}+\frac{\left(\sum_{t=1}^{4} x_{t}\right)^{2}}{2}+2 \sum_{t=1}^{4} x_{t}\right] \\
& \times d x_{1} \cdots d x_{4}=\frac{1}{16 \rho^{4}}\left(1-\gamma^{2}\right)^{-2}\left[-\frac{\sum_{t=1}^{4}\left(\alpha_{t}+1\right)^{-1}+3}{\prod_{t=1}^{4}\left(\alpha_{t}+1\right)}\right. \\
& \left.+\frac{1}{\prod_{t=1}^{4}\left(\alpha_{t}\right)}\left(3+\sum_{t=1}^{4} \frac{1}{\alpha_{t}^{2}}+\sum_{1 \leq t<p \leq 4} \frac{1}{\alpha_{t} \alpha_{p}}-\sum_{t=1}^{4} \frac{2}{\alpha_{t}}\right)\right]+o\left(\rho^{-4}\right),
\end{aligned}
$$

where a transmit correlation matrix $\mathbf{R}_{T}=\left[\begin{array}{ll}1 & \gamma \\ \gamma & 1\end{array}\right]$ is assumed.

\section{Simulation Results}

In this section, we examine the correctness of our theoretical analysis through computer simulation and study the influence of the transmit correlation on the error performance of USTM and RAS. To exploit the benefits of differential USTM, the optimized parametric codes in [1] are used in our simulations. For antenna selection, we select the best receive antenna based on the maximum received norm.

In Fig. 1, we compare the Chernoff bound on PEP presented in (26) with simulated PEP for a differential USTM system with $M=2, N=2$ when a single antenna is selected, $J=1$. Our theoretical bound is almost $1.5 \mathrm{~dB}$ away from the exact PEP at PEP equal to $10^{-5}$ for both the $\gamma=0.3$ and $\gamma=0.9$ cases and it gets tighter at a higher SNR. From Fig. 2, we observe that even for a high level of correlation, e.g. $\gamma=0.9$, although some loss occurs in the coding gain, both the fullcomplexity system and a system employing antenna selection exhibit the same diversity order $\left(G_{d}=4\right)$. To illustrate the advantage of RAS over no antenna selection subject to the same power consumption, we plot the performance of a system with a single receive antenna. As expected, its diversity order is equal to 2 .

\section{CONCLUSION}

In this paper, we analyzed the performance of USTM and RAS over the spatially-correlated Rayleigh fading channel. The popular exponential correlation model is used to describe the correlation among transmit antennas. The antenna selection performed at the receiver is based on the instantaneous received signal power. Our analysis uses the Chernoff bound on the PEP. The simulations and analysis showed that although the antenna correlation degrades the coding gain of the system, the diversity order remains the same as a that of the same system over an independent fading channel as long as the unitary 


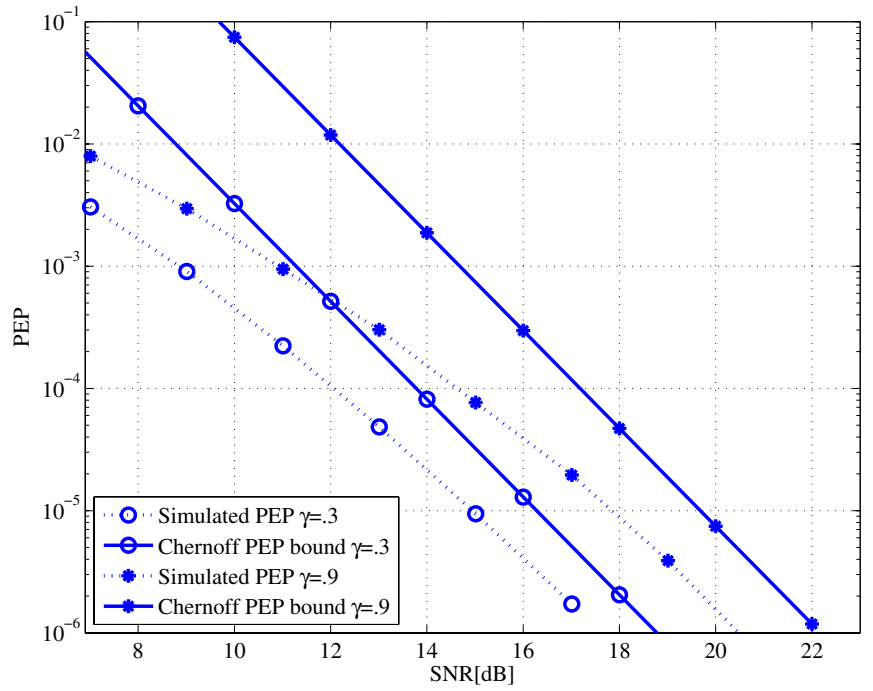

Fig. 1. Comparison of the Chernoff bound and the simulated PEP with $M=2, N=2 J=1$

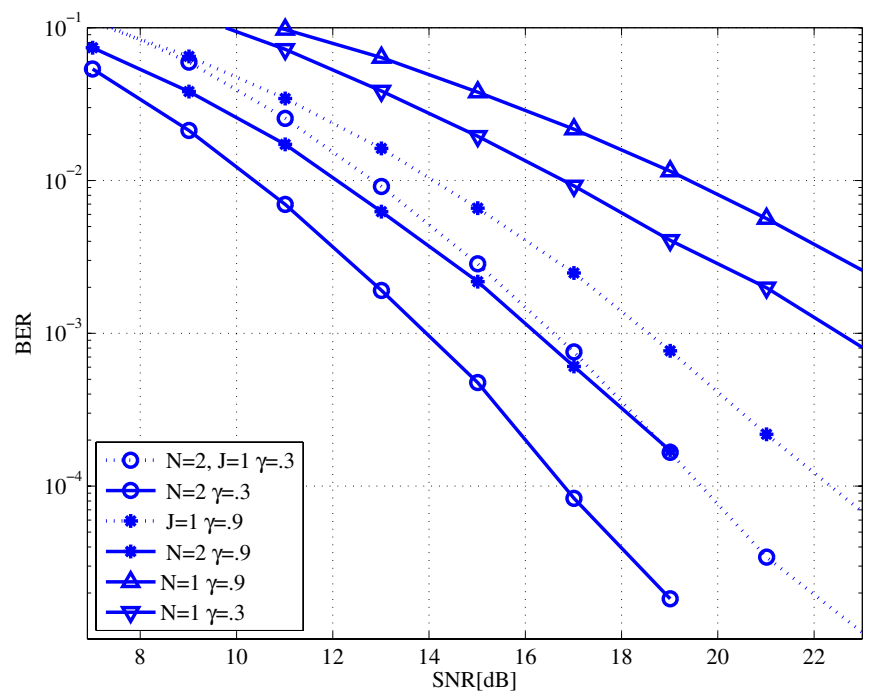

Fig. 2. Performance comparison of parametric codes for the system with or without antenna selection and $M=2, L=16$ over spatially correlated channel

signals are of full rank. For a simple $2 \times 2$ differential USTM system with single receive antenna selection, we presented a simple expression of the Chernoff bound and provided the simulation results.

\section{APPENDIX A}

PROOF OF THEOREM 2

By Induction theorem, let $f(s)=\left(\prod_{i=1}^{M}\left[1+s r_{i}\right]^{-1}\right)$. For $k=0$, it is clear that $\left.f(s)\right|_{s=-1}=C_{0} \rho^{-M}+o\left(\rho^{-M}\right)$, where
$C_{0}=(-M / T)^{M} \operatorname{det}^{-1}\left(\mathbf{R}_{T}\right)$. Similarly, for $k=1,2$, we have

$$
\begin{aligned}
& \left.f^{\prime}(s)\right|_{s=-1}=\sum_{i=1}^{M}-\left.r_{i}\left(1+r_{i} s\right)^{-1} f(s)\right|_{s=-1}=C_{1} \rho^{-M}+o\left(\rho^{-M}\right) \\
& \left.f^{\prime \prime}(s)\right|_{s=-1}=\left.\sum_{i=1}^{M} r_{i}^{2}\left(1+r_{i} s\right)^{-2} f(s)\right|_{s=-1}+\sum_{i=1}^{M}-r_{i}\left(1+r_{i} s\right)^{-1} \\
& \times\left. f^{\prime}(s)\right|_{s=-1}=C_{2} \rho^{-M}+o\left(\rho^{-M}\right),
\end{aligned}
$$

where $C_{1}=M C_{0}$, and $C_{2}=M(M+1) C_{0}$. Assuming that for all $k<n,\left.f^{(k)}(s)\right|_{s=-1}=C_{k} \rho^{-M}+o\left(\rho^{-M}\right)$, we show that for $k=n$ the same relation is held. i.e. $\left.f^{(n)}(s)\right|_{s=-1}=$ $C_{n} \rho^{-M}+o\left(\rho^{-M}\right)$. By using the same extension procedure used in (27), the $n$th derivative of $f(s)$ is expressed as a series of lower order derivatives:

$f^{(n)}(s)=\beta_{n} \sum_{i=1}^{M} r_{i}^{n}\left(1+r_{i} s\right)^{-n} f(s)+\cdots+\beta_{1} \sum_{i=1}^{M} r_{i}\left(1+r_{i} s\right)^{-1} f^{(n-1)}(s)$,

where $\beta_{1}, \ldots, \beta_{n}$ are independent of $\rho$ and can be obtained from: $\beta_{i}=(-1)^{i}(n-1) ! /(n-i)$ !. For any $k, C_{k}$ can be recursively calculated.

\section{REFERENCES}

[1] Q. Ma and C. Tepedelenlioglu, "Antenna selection for unitary space-time modulation," IEEE Trans. Inform. Theory, vol. 51, no. 10, pp. 3620 3631, Oct. 2005.

[2] B. Hochwald and T. Marzetta, "Unitary space-time modulation for multiple-antenna communications in Rayleigh flat fading," IEEE Trans. Inform. Theory, vol. 46, no. 2, pp. 543 - 564, Mar. 2000.

[3] C. Shan, A. Nallanathan, and P. Y. Kam, "A new class of signal constellations for differential unitary space-time modulation (DUSTM)," IEEE Commun. Lett., vol. 8, no. 1, pp. 1 - 3, 2004.

[4] I. Bahceci, T. Duman, and Y. Altunbasak, "Antenna selection for multiple-antenna transmission systems: performance analysis and code construction," in Proc. IEEE Int. Symp. on Infor. Theory (ISIT), Yokohama, Japan, 2003, pp. 93 -.

[5] D. Gore and A. Paulraj, "Space-time block coding with optimal antenna selection," in Proc. IEEE Int. Conf. Acoustics, Speech, and Signal Processing (ICASSP), vol. 4, Salt Lake City, UT, USA, 2001, pp. 2441 $-2444$.

[6] A. F. Molisch, M. Z. Win, and J. H. Winters, "Capacity of MIMO systems with antenna selection," in Proc. IEEE Int. Conf. Communications (ICC), vol. 2, Helsinki, Finland, 2001, pp. 570-574.

[7] J. Heath, R. W. and D. J. Love, "Multimode antenna selection for spatial multiplexing systems with linear receivers," IEEE Trans. Signal Processing, vol. 53, pp. 3042-3056, Aug. 2005.

[8] X. Cai and G. Giannakis, "Differential space-time modulation with eigen-beamforming for correlated MIMO fading channels," IEEE Trans. Signal Processing, vol. 54, no. 4, pp. 1279-1288, 2006.

[9] R. N. A. Paulraj and D. Gore, Introduction to Space-Time Wireless Communications, 1st ed. Cambridge University Press, 2003.

[10] B. Hochwald and W. Sweldens, "Differential unitary space-time modulation," IEEE Trans. Commun., vol. 48, no. 12, pp. 2041 - 2052, Dec. 2000.

[11] B. Holter and G. Oien, "On the amount of fading in MIMO diversity systems," IEEE Trans. Wireless Commun., vol. 4, no. 5, pp. 2498-2507, 2005.

[12] S. Kotz and J. Adams, "Distribution of sum of identically distributed exponentially correlated Gamma-variables."

[13] Z. Wang and G. B. Giannakis, "A simple and general parameterization quantifying performance in fading channels," IEEE Transactions on Communications, vol. 51, no. 8, pp. 1389-1398, Aug. 2003.

[14] M. Schwartz, W. R. Bennett, and S. Stein, Communication Systems and Techniques. New York: McGraw-Hill, 1966.

[15] R. A. Horn and C. R. Johnson, Matrix Analysis. Cambridge, U.K.: Cambridge Univ. Press, 1985. 